Curr Opin Rheumatol. 2012 November ; 24(6): 623-627. doi:10.1097/BOR.0b013e328358d59b.

\title{
Sarcopenia in older adults
}

\author{
Jeremy D. Walston \\ Division of Geriatric Medicine and Gerontology, Johns Hopkins University School of Medicine, \\ Baltimore, Maryland, USA
}

\begin{abstract}
Purpose of review-Sarcopenia, or the decline of skeletal muscle tissue with age, is one of the most important causes of functional decline and loss of independence in older adults. The purpose of this article is to review the current definitions of sarcopenia, its potential causes and clinical consequences, and the potential for intervention.
\end{abstract}

Recent findings-Although no consensus diagnosis has been reached, sarcopenia is increasingly defined by both loss of muscle mass and loss of muscle function or strength. Its cause is widely regarded as multifactorial, with neurological decline, hormonal changes, inflammatory pathway activation, declines in activity, chronic illness, fatty infiltration, and poor nutrition, all shown to be contributing factors. Recent molecular findings related to apoptosis, mitochondrial decline, and the angiotensin system in skeletal muscle have highlighted biological mechanisms that may be contributory. Interventions in general continue to target nutrition and exercise.

Summary-Efforts to develop a consensus definition are ongoing and will greatly facilitate the development and testing of novel interventions for sarcopenia. Although pharmaceutical agents targeting multiple biological pathways are being developed, adequate nutrition and targeted exercise remain the gold standard for therapy.

\section{Keywords}

aging; angiotensin system; inflammation; sarcopenia; sarcopenic obesity

\section{INTRODUCTION}

Sarcopenia has been defined as an age related, involuntary loss of skeletal muscle mass and strength. Beginning as early as the 4th decade of life, evidence suggests that skeletal muscle mass and skeletal muscle strength decline in a linear fashion, with up to $50 \%$ of mass being lost by the 8th decade of life [1]. Given that muscle mass accounts for up to $60 \%$ of body mass, pathological changes to this important metabolically active tissue can have profound consequences on the older adult. The consequences of sarcopenia are often severe in older adults, as the strength and functional declines associated with sarcopenia can in turn

(C) 2012 Wolters Kluwer Health | Lippincott Williams \& Wilkins

Correspondence to Jeremy D. Walston, MD, Johns Hopkins Asthma and Allergy Center, 5501 Hopkins Bayview Circle, Room 1A.62, Baltimore, MD 21217, USA. Tel: +1 410550 1003; fax: +410 550 3143; jwalston@jhmi.edu.

Conflicts of interest

There are no conflicts of interest. 
contribute to a number of adverse health outcomes, including loss of function, disability, and frailty [2,3,4"]. Sarcopenia is also associated with acute and chronic disease states, increased insulin resistance, fatigue, falls, and mortality [5-7]. Of the chronic disease states, sarcopenia has been especially associated with rheumatologic conditions, especially rheumatoid arthritis (RA) in women [8].

The physiological and morphological changes in skeletal muscle with advancing age are characterized by overall declines in size and number of skeletal muscle fibers, mainly the type 2 or fast-twitch muscle fibers, and a marked infiltration of fibrous and adipose tissue into the skeletal muscle [9]. In addition, satellite cells, the skeletal muscle precursor cells that reside in a quiescent state in association with myofibrils, likely also undergo important aging-related changes. These satellite cells are activated and begin the process of skeletal muscle repair and regeneration in response to the stress of heavy muscle use such as with weight-bearing activities or through traumatic events such as injury [10]. In the skeletal muscle of older adults, the satellite cell content is reduced and most specifically in the type-2 skeletal muscle fibers [11].

Although aging-related biological changes clearly drive sarcopenia, it is increasingly clear that obesity and fat infiltration into skeletal muscle play an important role in sarcopenia $[12,13]$. This phenomenon, termed sarcopenic obesity, is often grouped together with sarcopenia and likely plays an important role in a subset of those older adults thought to have sarcopenia. An analysis in the Framingham Study recently highlighted the functional and mobility limitations in sarcopenic obesity [2]. Investigators with the Health, Aging and Body Composition Study [14"], a longitudinal study of age-related changes in skeletal muscle and body composition in older adults, recently identified that increased fat mass was associated with lower muscle quality and an accelerated loss of lean body mass over 8 years.

The following review article briefly summarizes the ongoing debate over a clinical definition of sarcopenia, the multifactorial cause of sarcopenia, and the potential for intervention.

\section{CLINICAL DEFINITION OF SARCOPENIA}

A consensus clinical definition of sarcopenia or sarcopenic obesity does not presently exist. This is in part because of the lack of standardized measurements that capture functional skeletal muscle mass, because skeletal muscle mass measurements do not capture functionality, and because there has been no consensus in the research or clinical communities regarding its definition [15]. Many prior studies have used either muscle mass, muscle strength, or physical function measurements alone or in combination as markers for sarcopenia [16"]. In the past few years, investigators and clinicians have advocated for the inclusion of muscle strength or functional measurements related to strength such as grip strength and/or walking speed along with a muscle mass measurement in order to improve the diagnostic accuracy of sarcopenia [17]. Recent consensus conferences have been organized in order to move toward an acceptable clinical definition that can be utilized for both diagnosis and clinical research purposes. For example, the Society of Sarcopenia, Cachexia, and Wasting Disorders recently published a definition of sarcopenia with limited 
mobility that used a measure of walking speed and muscle mass [18]. The European Working Group on Sarcopenia in Older People (EWGSOP) recently recommended that measures of low muscle mass and low muscle strength or performance be utilized in any clinical definition [19]. This working group also has helped to provide important updates on currently available cut points for specific muscle-related measurements, and highlights the need for additional empirical data to be collected to facilitate the operationalization and validation of a sarcopenia diagnostic screening methodology that can be reproduced across many populations and integrated in Geriatrics practice.

\section{CAUSES OF SARCOPENIA}

The cause of sarcopenia is generally thought to be multifactorial, with environmental causes, disease triggers, inflammatory pathway activation, mitochondrial abnormalities, loss of neuromuscular junctions, reduced satellite cell numbers, and hormonal changes all thought to contribute (Fig. 1). In addition to these causes, recent progress in the understanding of molecular pathways that contribute to skeletal muscle maintenance has helped to further highlight Tissue growth factor (TGF)- $\beta$ signaling, apoptosis activation, and declines in mitochondrial function as potentially important in triggering sarcopenia as described in more detail below.

Environmental causes are usually divided between declines in activity and declines in nutritional intake. Older adults are less active, in part because of the increased chronic disease burden that leads to pain and fatigue [20]. In addition, declines in adequate protein and calorie intake, as well as overnutrition that results in sarcopenic obesity and accelerated loss of muscle mass and function, are important contributors to sarcopenia in older adults $\left[14^{*}, 21\right]$. Taken together, these environmental influences are superimposed on a multifactorial, age-related change in biology that pushes toward declines in skeletal muscle mass and strength as described below.

First, declines in the number of neuromuscular junctions, with resulting drop out of fasttwitch or type-II muscle fibers is thought to be play an important role in age-related muscle decline [22]. Recent studies in older female mice have demonstrated striking increases in the percentage of fully denervated neuromuscle junctions, especially in the fast-twitch muscle fibers such as in the extensor digitorum longus [23"]. Interestingly, the number of motor neurons in the spinal cord that innervate this area is not decreased, suggesting that this may be an axonal decline rather than a nerve body change per se. Because of the potential importance of this loss to the development of sarcopenia in humans, a potentially important new diagnostic screening measurement, the C-terminal agrin fragment, has been identified as a marker of neuromuscular junction decline in older men [24]. Second, declines in hormones that are important in muscle mass maintenance, including insulin-like growth factor-1, Dehydroepiandrosterone sulfate, testosterone, and estrogen, all likely contribute to sarcopenia $[25,26]$. These pathways also offer important potential opportunities for interventions [27]. Third, inflammatory pathway activation, likely due to a variety of disease and aging causes, is known to be an important contributor to sarcopenia [28]. A study [29] of serum levels of the inflammatory cytokine interleukin- 6 and its relationship to muscle strength in older women demonstrated a steeper decline in walking ability, a higher risk of 
developing physical disability, explained in part by a parallel decline in skeletal muscle strength. This may be in part due to the important influence of chronic inflammatory cytokine exposure on satellite cells in muscle fibers. The contribution of inflammation in older adults can come from multiple sources. Certainly, chronic disease states are among the most common triggers of inflammatory pathway activation. Many inflammatory rheumatological conditions such as systemic lupus erythematosus and RA are associated with muscle loss that is thought to be related to the chronic activation of inflammatory pathways that in turn negatively influence muscle regeneration [30"]. Many other chronic conditions, including renal failure and congestive heart failure, likely accelerate the development of sarcopenia via the increase of inflammatory mediators [31].

Finally, the aging-related loss of ability to replenish and replace skeletal muscle is increasingly evident. Skeletal muscle stem cells, crucial to the regeneration of skeletal muscle in older and younger adults, appear to be compromised in older adults in that they migrate at much slower speeds than younger cells, and their motility is hampered perhaps in part because of low levels of integrin expression [32"'].

\section{Newer molecular findings related to sarcopenia}

Underlying the more physiological causes outlined above are a host of age-related and disease-related biological changes that increase the vulnerability of older adults to the development of sarcopenia [16"]. Some important new studies have helped to shed light on aging-related molecular triggers for sarcopenia. Mitochondrial function and mitochondrial biogenesis appear to be altered in skeletal muscles of older adults, which in turn may contribute to altered skeletal muscle mass and function [33"']. Aging-related changes in the angiotensin system were recently identified to play a crucial role in skeletal muscle healing and in disuse atrophy of skeletal muscle [34"']. Importantly, in this same study, the angiotensin type-1 receptor blocking agent losartan was found to help accelerate skeletal muscle healing and prevent disuse atrophy in treated older mice, likely in response to downregulation of the TGF- $\beta$ pathways. Losartan was also demonstrated to increase the number of angiotensin type-2 receptors in the mitochondria of older mice, which in turn explains some of the improved skeletal muscle healing that was observed in older mice treated with losartan [35"]. Apoptosis, or programmed cell death may also play an important role in sarcopenia development. Preliminary studies of proteins associated with apoptosis showed an upregulation in those older adults with lower thigh muscle volume and slower gait speed, suggesting that apoptosis may contribute to this muscle decline. The apoptosisinducing factor, usually located in the intermitochondrial membrane space, was recently found to protect skeletal muscle precursors, or satellite cells, from apoptosis so long as it was not released into the cytoplasm of cells [36]. Finally, newer evidence suggests that altered transcriptional regulation of mRNA and translation of proteins negatively impact proteins important in myogenesis, including Pax 7, myogenin, and MyoD [37].

\section{Interventions for sarcopenia}

Although substantial progress has been made in the understanding of the multifactorial causes of sarcopenia, most interventions have focused on improving the environmental causes of sarcopenia, namely through increasing activity and providing adequate nutrition. 
Given the high prevalence of sarcopenia, and close relationship to fatigue, functional decline, and chronic illness, the development of interventions has been touted by drug companies and investigators as a crucially important target for intervention in older adults [38]. Although a large-scale clinical trial that targets the prevention or treatment of sarcopenia per se has not been attempted, many studies have indirectly targeted sarcopenia by using age-related declines in physical function as an outcome. Results of clinical intervention studies in even the oldest and frailest nursing home residents have demonstrated significant functional improvement through a combination of nutrition and resistance exercise [39]. Recent clinical studies continue to show this with significant increases in muscle protein synthesis in older adults who receive physical activity and nutrition [40"]. Other groups continue to refine the best exercise modality to treat or prevent sarcopenia $\left[10,16^{*}, 41\right]$. These interventions appear to positively impact satellite cell dysfunction, neuromuscular junction decline, and mitochondrial biogenesis. Although endocrine interventions targeting muscle function and strength have been developed previously, few, if any, have shown efficacy [26]. Future pharmaceutical interventions for sarcopenia will also likely target very specific molecular pathways such as the angiotensin system, apoptosis, and mitochondrial function.

In order to develop the next generation of studies targeting sarcopenia per se, it will be important to develop a consensus definition that can be utilized across a number of studies and populations in order to determine safety and efficacy for sarcopenia. The International Working Group on Sarcopenia has developed recommendations on the design of phase IIB clinical trials and cautions that appropriate muscle mass measurements and physical function measurements, along with sufficient periods of time, are necessary to adequately test any proposed pharmaceutical intervention [42]. EWGSOP also strongly recommends that measures of muscle mass, muscle strength, and/or functional performance be utilized in any clinical definition [19], which will help to facilitate the operationalization and validation of a sarcopenia diagnostic screening methodology that can be reproduced across many populations, used in clinical trials, and integrated into the practice of geriatric medicine.

\section{CONCLUSION}

Sarcopenia remains an important clinical problem that impacts millions of older adults. Despite its high prevalence, no clear consensus definition has been developed. Causes include declines in hormones and numbers of neuromuscular junctions, increased inflammation, declines in activity, and inadequate nutrition. Newer molecular discoveries that may impact sarcopenia include alterations in mitochondrial biology, the angiotensin system, and apoptosis. Interventions for sarcopenia continue to be developed with most emphasis on exercise and nutritional interventions. Pharmaceutical development has in part been slowed by the lack of a consensus definition for sarcopenia.

\section{REFERENCES AND RECOMMENDED READING}

Papers of particular interest, published within the annual period of review, have been highlighted as:

- of special interest 
- of outstanding interest

Additional references related to this topic can also be found in the Current World Literature section in this issue (pp. 695-697).

1. Metter EJ, Conwit R, Tobin J, Fozard JL. Age-associated loss of power and strength in the upper extremities in women and men. J Gerontol A Biol Sci Med Sci. 1997; 52:B267-B276. [PubMed: 9310077]

2. Dufour AB, Hannan MT, Murabito JM, et al. Sarcopenia definitions considering body size and fat mass are associated with mobility limitations: The Framingham Study. J Gerontol A Biol Sci Med Sci. 2012 Epub ahead of print.

3. Xue QL, Walston JD, Fried LP, Beamer BA. Prediction of risk of falling, physical disability, and frailty by rate of decline in grip strength: the women's health and aging study. Arch Intern Med. 2011; 171:1119-1121. [PubMed: 21709116]

4-. Marsh AP, Rejeski WJ, Espeland MA, et al. Muscle strength and BMI as predictors of major mobility disability in the Lifestyle Interventions and Independence for Elders pilot (LIFE-P). J Gerontol A Biol Sci Med Sci. 2011; 66:1376-1383. This article describes how both strength and body mass are important predictors of disability in older adults. [PubMed: 21975090]

5. Peng P, Hyder O, Firoozmand A, et al. Impact of sarcopenia on outcomes following resection of pancreatic adenocarcinoma. J Gastrointest Surg. 2012; 16:1478-1486. [PubMed: 22692586]

6. Newman AB, Kupelian V, Visser M, et al. Strength, but not muscle mass, is associated with mortality in the health, aging and body composition study cohort. J Gerontol A Biol Sci Med Sci. 2006; 61:72-77. [PubMed: 16456196]

7. Landi F, Liperoti R, Russo A, et al. Sarcopenia as a risk factor for falls in elderly individuals: Results from the ilSIRENTE study. Clin Nutr. 2012 Epub ahead of print.

8. Giles JT, Ling SM, Ferrucci L, et al. Abnormal body composition phenotypes in older rheumatoid arthritis patients: association with disease characteristics and pharmacotherapies. Arthritis Rheum. 2008; 59:807-815. [PubMed: 18512711]

9. Lexell J. Human aging, muscle mass, and fiber type composition. J Gerontol A Biol Sci Med Sci. 1995; 50:11-16. [PubMed: 7493202]

10. Snijders T, Verdijk LB, van Loon LJ. The impact of sarcopenia and exercise training on skeletal muscle satellite cells. Ageing Res Rev. 2009; 8:328-338. [PubMed: 19464390]

11. Verdijk LB, Koopman R, Schaart G, et al. Satellite cell content is specifically reduced in type II skeletal muscle fibers in the elderly. Am J Physiol Endocrinol Metab. 2007; 292:E151-E157. [PubMed: 16926381]

12. Prado CM, Wells JC, Smith SR, et al. Sarcopenic obesity: a critical appraisal of the current evidence. Clin Nutr. 2012 Epub ahead of print.

13. Marcus RL, Brixner DI, Ghate S, Lastayo P. Fat modulates the relationship between sarcopenia and physical function in nonobese older adults. Curr Gerontol Geriatr Res. 2012; 2012:216185. [PubMed: 22312329]

14-. Koster A, Ding J, Stenholm S, et al. Does the amount of fat mass predict agerelated loss of lean mass, muscle strength, and muscle quality in older adults? J Gerontol A Biol Sci Med Sci. 2011; 66:888-895. This study demonstrates the important contribution of fat to measurements of sarcopenia in older adults. [PubMed: 21572082]

15. Bijlsma AY, Meskers CG, Ling CH, et al. Defining sarcopenia: the impact of different diagnostic criteria on the prevalence of sarcopenia in a large middle aged cohort. Age (Dordr). 2012 Epub ahead of print.

16-. Ferrucci L, de Cabo FR, Knuth ND, Studenski S. Of Greek heroes, wiggling worms, mighty mice, and old body builders. J Gerontol A Biol Sci Med Sci. 2012; 67:13-16. This review study highlights important current insights into the epidemiology, causes, and diagnostic dilemmas that accompany sarcopenia research. [PubMed: 22113943]

17. Bellan van KG, Houles M, Vellas B. Identifying sarcopenia. Curr Opin Clin Nutr Metab Care. 2012 Epub ahead of print. 
18. Morley JE, Abbatecola AM, Argiles JM, et al. Sarcopenia with limited mobility: an international consensus. J Am Med Dir Assoc. 2011; 12:403-409. [PubMed: 21640657]

19. Cruz-Jentoft AJ, Baeyens JP, Bauer JM, et al. Sarcopenia: European consensus on definition and diagnosis: report of the European Working Group on Sarcopenia in Older People. Age Ageing. 2010; 39:412-423. [PubMed: 20392703]

20. Marquez DX, Hoyem R, Fogg L, et al. Physical activity of urban community-dwelling older Latino adults. J Phys Act Health. 2011; 8 (Suppl 2):S161-S170. [PubMed: 21918229]

21. Robinson S, Cooper C, Aihie SA. Nutrition and sarcopenia: a review of the evidence and implications for preventive strategies. J Aging Res. 2012; 2012:510801. [PubMed: 22506112]

22. Verdijk LB, Dirks ML, Snijders T, et al. Reduced satellite cell numbers with spinal cord injury and aging in humans. Med Sci Sports Exerc. 2012 Epub ahead of print.

23-. Chai RJ, Vukovic J, Dunlop S, et al. Striking denervation of neuromuscular junctions without lumbar motoneuron loss in geriatric mouse muscle. PLoS One. 2011; 6:e28090. This article describes the loss of innervation of muscle in older adults and the preservation of the motor neuron, which may help highlight a mechanistic cause of sarcopenia in future studies. [PubMed: 22164231]

24. Drey M, Sieber CC, Bauer JM, et al. C-terminal agrin fragment as a potential marker for sarcopenia caused by degeneration of the neuromuscular junction. Exp Gerontol. 2012 Epub ahead of print.

25. McIntire KL, Hoffman AR. The endocrine system and sarcopenia: potential therapeutic benefits. Curr Aging Sci. 2011; 4:298-305. [PubMed: 21529322]

26. Sakuma K, Yamaguchi A. Sarcopenia and age-related endocrine function. Int J Endocrinol. 2012; 2012:127362. [PubMed: 22690213]

27. Horstman AM, Dillon EL, Urban RJ, Sheffield-Moore M. The role of androgens and estrogens on healthy aging and longevity. J Gerontol A Biol Sci Med Sci. 2012 Epub ahead of print.

28. Jo E, Lee SR, Park BS, Kim JS. Potential mechanisms underlying the role of chronic inflammation in age-related muscle wasting. Aging Clin Exp Res. 2012 Epub ahead of print.

29. Ferrucci L, Penninx BW, Volpato S, et al. Change in muscle strength explains accelerated decline of physical function in older women with high interleukin-6 serum levels. J Am Geriatr Soc. 2002; 50:1947-1954. [PubMed: 12473005]

30-. Santos MJ, Vinagre F, Canas da SJ, et al. Body composition phenotypes in systemic lupus erythematosus and rheumatoid arthritis: a comparative study of Caucasian female patients. Clin Exp Rheumatol. 2011; 29:470-476. This study describes the associations between these common rheumatological disease states and increased fat mass and loss of lean mass. [PubMed: 21640047]

31. Domanski M, Ciechanowski K. Sarcopenia: a major challenge in elderly patients with end-stage renal disease. J Aging Res. 2012; 2012:754739. [PubMed: 22536505]

32-r. Collins-Hooper H, Woolley TE, Dyson L, et al. Age-related changes in speed and mechanism of adult skeletal muscle stem cell migration. Stem Cells. 2012; 30:1182-1195. This study demonstrates how muscle stem cells' motility and migratory ability decline with aging. [PubMed: 22593017]

33-. Joseph AM, Adhihetty PJ, Buford TW, et al. The impact of aging on mitochondrial function and biogenesis pathways in skeletal muscle of sedentary high and low-functioning elderly individuals. Aging Cell. 2012 Epub ahead of print. This study highlights a crucial potential etiological pathway that warrants further investigation.

34-. Burks TN, Andres-Mateos E, Marx R, et al. Losartan restores skeletal muscle remodeling and protects against disuse atrophy in sarcopenia. Sci Transl Med. 2011; 3:82ra37. This study highlights the angiotensin system as crucially important in aging skeletal muscle. Importantly, it provides a potential pathway by which skeletal muscle decline can be reversed.

35-. Abadir PM, Foster DB, Crow M, et al. Identification and characterization of a functional mitochondrial angiotensin system. Proc Natl Acad Sci U S A. 2011; 108:14849-14854. This article highlights a newly discovered mitochondrial angiotensin system that is present in muscle and that is markedly downregulated in aging, suggesting that it may be important for futuretargeted interventions for sarcopenia. [PubMed: 21852574] 
36. Armand AS, Laziz I, Djeghloul D, et al. Apoptosis-inducing factor regulates skeletal muscle progenitor cell number and muscle phenotype. PLoS One. 2011; 6:e27283. [PubMed: 22076146]

37. Ma JF, Hall DT, Gallouzi IE. The impact of mRNA turnover and translation on age-related muscle loss. Ageing Res Rev. 2012 Epub ahead of print.

38. Matthews GD, Huang CL, Sun L, Zaidi M. Translational musculoskeletal science: is sarcopenia the next clinical target after osteoporosis? Ann N Y Acad Sci. 2011; 1237:95-105. [PubMed: 22082371]

39. Fiatarone MA, O'Neill EF, Ryan ND, et al. Exercise training and nutritional supplementation for physical frailty in very elderly people. N Engl J Med. 1994; 330:1769-1775. [PubMed: 8190152]

40-. Timmerman KL, Dhanani S, Glynn EL, et al. A moderate acute increase in physical activity enhances nutritive flow and the muscle protein anabolic response to mixed nutrient intake in older adults. Am J Clin Nutr. 2012; 95:1403-1412. This study demonstrates the importance of exercise and activity in getting nutrition into skeletal muscle, which supports the use of both activity and nutrition as important interventions for sarcopenia. [PubMed: 22572647]

41. Aagaard P, Suetta C, Caserotti P, et al. Role of the nervous system in sarcopenia and muscle atrophy with aging: strength training as a countermeasure. Scand J Med Sci Sports. 2010; 20:4964. [PubMed: 20487503]

42. Chumlea WC, Cesari M, Evans WJ, et al. Sarcopenia: designing phase IIB trials. J Nutr Health Aging. 2011; 15:450-455. [PubMed: 21623466] 


\section{KEY POINTS}

- Sarcopenia is a common condition in older adults that contributes to functional decline, disability, frailty, and falls.

- There is as of yet no consensus definition for sarcopenia, although recommendations for the development of a definition based on both muscle mass measurement and physical function have been proposed.

- Sarcopenia has a multifactorial cause, with declines in activity and nutrition, disease states, inflammation, declines in neuromuscular junctions, and aging related changes in mitochondria, apoptosis, and the angiotensin system recently found to be contributory.

- Rheumatological conditions are highly associated with sarcopenia/skeletal muscle mass decline, likely due to the high levels of inflammatory cytokines.

- Clinical interventions have focused on exercise and nutrition, with pharmaceutical testing lagging in part because of the lack of a consensus definition. 


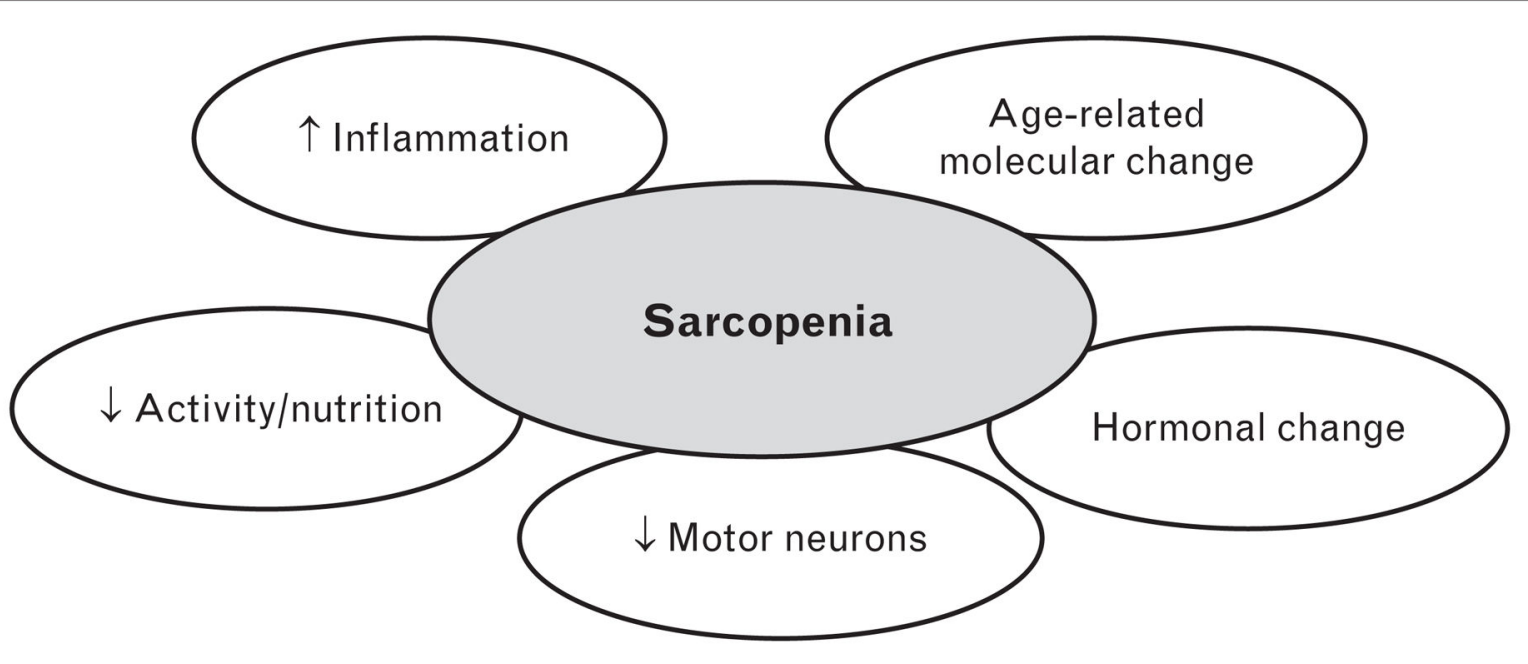

FIGURE 1.

Multifactorial cause of sarcopenia. The ovals represent domains known to influence the maintenance of skeletal muscle strength and mass in aging organisms. 